\title{
A Comprehensive Model for Decision-making Problem Incorporating Uncertainty
}

\section{Persis Voola}

University College of Engineering, Adikavi Nannaya University, Rajamahendravaram, Andhra Pradesh, India Corresponding author: persisvoola.cse@aknu.edu.in

Received: 11 Mar., 2021

Revised: 17 May, 2021

Accepted: 10 Jun., 2021

\begin{abstract}
Decision-making is an assessment activity where several alternatives will be assessed to identify the best alternative or the optimal set of alternatives. The assessment that is carried out by humans is always not precise. Imprecision may exist in the form of incompleteness, ignorance, vagueness and uncertainty. The literature records many techniques for decision-making, which are based on different scales of assessment. Further, there are algorithms which aggregate assessments data for meaningful results and analyses. The objective of this paper is to present a comprehensive model which integrates collection of assessments incorporating uncertainty and aggregation of the obtained data to generate ranks of preference of the alternatives. Interested researchers can envisage the model in different empirical and contextual settings to extract the potential.
\end{abstract}

Keywords: Decision-making, uncertainty, interval scale, aggregation

Assessment is a decision-making problem that helps to establish priorities or ranks. Priorities come into picture when several alternatives exist and the best alternative or the optimal set of alternatives has to be picked depending on the decision problem. For example, in the context of an educational institute where an academic administrator has to be appointed, the best candidature has to be selected out of a list of candidatures where as in the context of software development one need to assess the priorities of requirements for implementation in order to identify the optimal set of requirements that can be implemented with the available resources.

The central theme of any assessment is uncertainty. Raanan Lipshitz, 1997 et al. ${ }^{[1]}$ performed study of 102 reports related to assessment and noted that incomplete information, inadequate understanding and undifferentiated alternatives contribute for uncertainty. This establishes the ground that assessment has to incorporate incomplete and uncertain nature of human judgment. The decision-making literature 
ascertains ambiguity coupled with uncertainty as a recognized concept ${ }^{[2,3]}$. This is substantiated by the fact that assessors are humans whose assessment is more likely to be grounded on opinions, experience, intelligence, assumptions and beliefs. As a result, it is more likely that an assessor is comfortable with intervals or ranges for assessment rather than precise values or grades.

Section 2 presents the contextual setting of the comprehensive model. Section 3 presents different forms of carrying assessment using assessment scales. Section 4 presents the comprehensive model. Section $\mathrm{V}$ presents the conclusion.

\section{Contextual Setting of the Comprehensive Model}

The comprehensive model presented in this paper conforms to Multi Criteria Decision-Making (MCDM) problems. MCDM problems are those that assess alternatives influenced by multiple criteria. MCDM problems are found commonly in simple to complex every day decisions, sciences, engineering and business domains.

MCDM problem is described by Keeney and Raiffa $(1976)^{[4]}$ in the following way:

$\square$ A set of decision alternatives for evaluation

$\square$ A set of criteria along which the alternatives are to be evaluated

$\square$ The ranking of alternatives in the order of preference.

The first two elements of MCDM comprise the inputs and the third element is the output of the problem. These three features are well accepted in the literature on decision-making. Eom, 1989 ${ }^{[5]}$ records the applicability of MCDM models in a wide range of domains: employee appraisal, resource allocation, organization evaluation, engineering design evaluation, supplier evaluation, urban and community planning.

The decision problem has to be thoroughly understood in the light of the features of an MCDM problem. The alternatives to be assessed and the criteria along which they have to be assessed have to be identified. The number of assessors who assess the alternatives and their relative weights has to be formulated. The description of the assessment scale with relevant grades has to be chosen. This defines the contextual setting of the comprehensive model.

\section{Scales of Assessment}

Stevens, 1946 in his work "On the Theory of Scales of Measurement"[6] proposed four scales of measurement: Nominal, Ordinal, Interval and Ratio scales. A comparative study of the scales of measurement in the context of software requirements is conducted as an experiment by the author and the results can be found $\mathrm{in}^{[7]}$. A brief description of the scales is presented below:

\section{(a) Nominal Scale}

This is the categorical scale where the alternatives to be assessed will be placed under one of the categories. For example, the performance of a student in a cultural event can be categorized as Excellent, Very Good, Good and Poor. 


\section{(b) Ordinal Scale}

This is the ordering scale where the alternatives to be assessed will be ranked from 1 to $n$. For example, based on the total marks gained by a student in the exams, they can be ranked from 1 to $n$, where rank 1 denotes the highest total marks and rank $n$ denotes the lowest total marks.

\section{(c) Ratio Scale}

This is the scale with ratios where the alternatives to be assessed will be compared pairwise and processed mathematically to derive the final priorities. The usage and applications of ratio scale are discussed at length in ${ }^{[8]}$.

\section{(d) Interval Scale}

Interval scale is the choice of interest because it addresses incorporation of uncertainty during assessment whereas the other three scales are based on precise values. This is the scale with ranges of values where the alternatives to be assessed will be assigned to intervals i.e., range of values rather than a precise value. For example, marks scored between 90 and 100 will be assigned to the grade $\mathrm{A}+$ whereas marks scored between 80 and 89 will be assigned to the grade $\mathrm{A}$ and so on.

\section{Comprehensive Model}

The model presented in this section addresses how uncertainty of assessments can be incorporated and how the collected imprecise assessments can be aggregated to generate precise values. The model begins with initial setup: identification of assessors, alternatives, grades of interval scale and the criteria along which the alternatives have to be assessed. Then it moves to the collection of assessments followed by aggregation of the collected assessments.

\section{(a) Initial Setup of the Model}

The initial setup of the model comprises the following activities:

\section{Identification of the assessors}

Assessors are human decision makers who are interested in the outcome of the product or process. Competing and conflicting personal preferences exist among the assessors. An individual assessor or representative participation or a complete set of assessors may be chosen depending on the context of the problem. However, in many real time situations group assessment is recommended over an individual's because of its property of less prone to error ${ }^{[9]}$. Further, it has to be noted that all assessors may not be equally contributing for the outcome of the product. In such cases, they have to be assigned with relative weights.

\section{Identification of the alternatives}

Alternatives are the objects of the problem under study. They can range from 2 to $n$. Generally, $n$ will be in tens and in some cases it may be in hundreds. The description of the alternatives should be clear and 
understandable consistently among all assessors. Redundant, irrelevant and missing alternatives should be addressed.

\section{Identification of the grades of interval scale}

If there are $\mathrm{n}$ precise grades, then the total number of scales on interval scale will be $n(n+1) / 2$, for $n=$ $2,3,4$. In real time, $n$ value beyond 4 may not be useful as the number of grades with $n=5$ is 16 .

For example, if the grades identified for assessment are Poor(P), Average(A), Good(G) and Excellent(E) then interval scale grades will be P, A, G, E, PA, PG, AG, AE, GE, PE, where single letters stand for precise grades and double letters stand for interval grades. For example, AE stands for the range between Average and Excellent.

\section{Identify the criteria along which the alternatives have to be assessed}

Assessment of alternatives is not a straightforward decision as multiple criteria affecting the alternatives need to be taken into consideration. The selection of criteria has to be done carefully such that they are relevant to the decision problem under consideration, and their interpretation is consistent among all assessors. For example, in order to identify the best student of a class, total score of marks is not the only criteria, but several criteria like badges earned through certifications, participation in academic events, contribution to research and consultancy, performance in extracurricular activities etc to be considered.

\section{(b) Collect the Assessments}

Let the alternatives to be assessed are $A_{i}, i=2$ to $n$. Let $G$ represent the set of individual and interval grades used during assessment represented as $G=\left\{G_{1}, G_{2}, G_{3}, G_{12}, G_{23}, G_{13}\right\}$ where $G_{1}, G_{2}, G_{3}$ are the individual grades from lowest to highest and $G_{12}, G_{23}, G_{13}$ are interval grades. Let the human assessors be $H_{x}, H_{y}$. Then their assessment of an alternative $A_{i}$ to the grades in $G$ will be as follows.

$$
\begin{aligned}
& H_{x}\left(A_{i}\right)=\left\{\left(G_{1}, g_{1 x} \%\right),\left(G_{2}, g_{2 x} \%\right),\left(G_{3}, g_{3 x} \%\right),\left(G_{12}, g_{12 x} \%\right),\left(G_{23}, g_{23 x} \%\right),\left(G_{13}, g_{13 x} \%\right)\right\} \\
& H_{y}\left(A_{i}\right)=\left\{\left(G_{1}, g_{1 y} \%\right),\left(G_{2}, g_{2 y} \%\right),\left(G_{3}, g_{3 y} \%\right),\left(G_{12}, g_{12 y} \%\right),\left(G_{23}, g_{23 y} \%\right),\left(G_{13}, g_{13 y} \%\right)\right\}
\end{aligned}
$$

The first element in each set represents individual grades and subsets of adjacent grades and the second element in each set represents degree of belief associated with that grade expressed in percentage. The total degrees of belief should be 1 .

\section{(c) Aggregate the Assessments}

Once assessments are collected, they should be aggregated. Interval Evidential Reasoning (IER) ${ }^{[10,11]}$ is an established algorithm in the literature that is based on Evidential Reasoning ${ }^{[12]}$. The inputs to IER are modelled as shown in (1) and (2). This conforms to the Belief Decision Matrix (BDM) for problem modelling of IER. A brief description of the algorithm is presented below.

Let $w_{x}$ and $w_{y}$ be the normalized weights of $H_{x}$ and $H_{y}$. Weight assignment method as described in ${ }^{[13]}$ can be used to assign weights to the assessors. Basic probability masses are computed as the product of the 
assessor weight and the degree of belief corresponding to each grade. These are placed across and along the first row and column of Table 1 for $H_{x}$ and $H_{y}$ respectively.

Table 1: Basic Probability Masses

\begin{tabular}{llllllll}
\hline$H_{x} \oplus$ & $m x g_{2}:$ & $m x g_{2}:$ & $m x g_{3}:$ & $m x g_{12}:$ & $m x g_{23}:$ & $m x g_{13}:$ & $m_{x G}:$ \\
$H_{y}$ & $w_{x} g_{2}$ & $w_{x} g_{2}$ & $w_{x} g_{3}$ & $w_{x} g_{12}$ & $w_{x} g_{23}$ & $w_{x} g_{13}$ & $1-w_{x}$ \\
\hline$m y g_{1}:$ & $m x g_{1} m y g_{1}$ & $m x g_{2} m y g_{1}$ & $m x g_{3} m y g_{1}$ & $m x g_{12} m y g_{1}$ & $m x g_{23} m y g_{1}$ & $m x g_{13} m y g_{1}$ & $m x G_{m} y g_{1}$ \\
$w_{y} g_{1}$ & $\left\{G_{1}\right\}$ & $\{\varphi\}$ & $\{\varphi\}$ & $\left\{\mathrm{G}_{1}\right\}$ & $\{\varphi\}$ & $\left\{\mathrm{G}_{1}\right\}$ & $\left\{\mathrm{G}_{1}\right\}$ \\
$m y g_{2}:$ & $m x g_{1} m y g_{2}$ & $m x g_{2} m y g_{2}$ & $m x g_{3} m y g_{2}$ & $m x g_{12} m y g_{2}$ & $m x g_{23} m y g_{2}$ & $m x g_{13} m y g_{2}$ & $m x G_{m} y g_{2}$ \\
$w_{y} g_{2}$ & $\{\varphi\}$ & $\left\{\mathrm{G}_{2}\right\}$ & $\{\varphi\}$ & $\left\{\mathrm{G}_{2}\right\}$ & $\left\{\mathrm{G}_{2}\right\}$ & $\left\{\mathrm{G}_{2}\right\}$ & $\left\{\mathrm{G}_{2}\right\}$ \\
$m y g_{3}:$ & $m x g_{1} m y g_{3}$ & $m x g_{2} m y g_{3}$ & $m x g_{3} m y g_{3}$ & $m x g_{12} m y g_{3}$ & $m x g_{23} m y g_{3}$ & $m x g_{13} m y g_{3}$ & $m x G_{m} y g_{3}$ \\
$w_{y} g_{3}$ & $\{\varphi\}$ & $\{\varphi\}$ & $\left\{\mathrm{G}_{3}\right\}$ & $\{\varphi\}$ & $\left\{\mathrm{G}_{3}\right\}$ & $\left\{\mathrm{G}_{3}\right\}$ & $\left\{\mathrm{G}_{3}\right\}$ \\
$m y g_{1}:$ & $m x g_{1} m y g_{12}$ & $m x g_{2} m y g_{12}$ & $m x g_{3} m y g_{12}$ & $m x g_{12} m y g_{12}$ & $m x g_{23} m y g_{12}$ & $m x g_{13} m y g_{12}$ & $m x G_{m} y g_{12}$ \\
$w_{y} g_{12}$ & $\left\{\mathrm{G}_{1}\right\}$ & $\left\{\mathrm{G}_{2}\right\}$ & $\{\varphi\}$ & $\left\{\mathrm{G}_{12}\right\}$ & $\left\{\mathrm{G}_{2}\right\}$ & $\left\{\mathrm{G}_{12}\right\}$ & $\left\{\mathrm{G}_{12}\right\}$ \\
$m y g_{2}:$ & $m x g_{1} m y g_{23}$ & $m x g_{2} m y g_{23}$ & $m x g_{3} m y g_{23}$ & $m x g_{12} m y g_{23}$ & $m x g_{23} m y g_{23}$ & $m x g_{13} m y g_{23}$ & $m x G_{m} y g_{23}$ \\
$w_{y} g_{23}$ & $\{\varphi\}$ & $\left\{\mathrm{G}_{2}\right\}$ & $\left\{\mathrm{G}_{3}\right\}$ & $\left\{\mathrm{G}_{2}\right\}$ & $\left\{\mathrm{G}_{23}\right\}$ & $\left\{\mathrm{G}_{23}\right\}$ & $\left\{\mathrm{G}_{23}\right\}$ \\
$m y g_{1}:$ & $m x g_{1} m y g_{13}$ & $m x g_{2} m y g_{13}$ & $m x g_{3} m y g_{13}$ & $m x g_{12} m y g_{13}$ & $m x g_{23} m y g_{13}$ & $m x g_{13} m y g_{13}$ & $m x G_{m} y g_{13}$ \\
$w_{y} g_{13}$ & $\left\{\mathrm{G}_{1}\right\}$ & $\left\{\mathrm{G}_{2}\right\}$ & $\left\{\mathrm{G}_{3}\right\}$ & $\left\{\mathrm{G}_{12}\right\}$ & $\left\{\mathrm{G}_{23}\right\}$ & $\left\{\mathrm{G}_{13}\right\}$ & $\left\{\mathrm{G}_{13}\right\}$ \\
$m y g:$ & $m x g_{1} m y G$ & $m x g_{2} m y G$ & $m x g_{3} m y G$ & $m x g_{12} m y G$ & $m x g_{12} m y G$ & $m x g_{13} m y G$ & $m x G_{m} y G$ \\
$1-w_{y}$ & $\left\{\mathrm{G}_{1}\right\}$ & $\left\{\mathrm{G}_{2}\right\}$ & $\left\{\mathrm{G}_{3}\right\}$ & $\left\{\mathrm{G}_{12}\right\}$ & $\left\{\mathrm{G}_{23}\right\}$ & $\left\{\mathrm{G}_{13}\right\}$ & \\
\hline
\end{tabular}

The last cell of the first row and the first column denote the remaining probability mass that is to be assigned based on the significance of other assessors. The cells of the table are used for summing up the probability mass values assigned to an individual grade or interval grade as follows:

$$
\begin{aligned}
& S_{G_{1}}=\frac{1}{1-c} \times \text { sum of probability mass values of } G_{1} \\
& S_{G_{2}}=\frac{1}{1-c} \times \text { sum of probability mass values of } G_{2} \\
& S_{G_{3}}=\frac{1}{1-c} \times \text { sum of probability mass values of } G_{3} \\
& S_{G_{12}}=\frac{1}{1-c} \times \text { sum of probability mass values of } G_{12} \\
& S_{G_{23}}=\frac{1}{1-c} \times \text { sum of probability mass values of } G_{23} \\
& S_{G_{13}}=\frac{1}{1-c} \times \text { sum of probability mass values of } G_{13}
\end{aligned}
$$

Then probability mass at large in $G$ is calculated as:

$$
S_{G}=\frac{1}{1-c} \times \text { sum of probability mass values of } G
$$

The sum of all probability masses pertaining to the empty set $\omega$ is assigned to $c$. 
The scaling factor $\frac{1}{1-c}$ is to ensure

$$
S_{G_{1}}+S_{G_{2}}+S_{G_{3}}+S_{G_{12}}+S_{G_{23}}+S_{G_{13}}+S_{G}=1
$$

The combined probability masses arrived at finally are shown in (3) to (8). The aggregated belief degrees are calculated from these values by assigning $S_{G}$ back to all grades of the scale in proportion as shown in (11) to (16).

$$
\begin{aligned}
& g_{1 x y}=\frac{S_{G_{1}}}{1-C_{G}} \\
& g_{2 x y}=\frac{S_{G_{2}}}{1-C_{G}} \\
& g_{3 x y}=\frac{S_{G_{3}}}{1-C_{G}} \\
& g_{12 x y}=\frac{S_{G_{12}}}{1-C_{G}} \\
& g_{23 x y}=\frac{S_{G_{23}}}{1-C_{G}} \\
& g_{13 x y}=\frac{S_{G_{13}}}{1-C_{G}}
\end{aligned}
$$

Aggregated assessment of $H_{x}, H_{y}$ can now be expressed with aggregated belief degrees of (11) to (16) as shown below.

$$
H_{x y}(A)=\left\{\left(G_{1}, g_{1 x y} \%\right)\left(G_{2}, g_{2 x y} \%\right)\left(G_{3}, g_{3 x y} \%\right)\left(G_{12}, g_{12 x y} \%\right)\left(G_{23}, g_{23 x y} \%\right)\left(G_{13}, g_{13 x y} \%\right)\right\}
$$

The ranks of preference of the alternatives are not visible from the aggregated belief degrees. The following steps are used to generate ranks.

The utilities of the grades have to be estimated first, to precisely rank the requirements. Utility value can be estimated using probability method or Regression model $\mathbf{S}^{[14]}$. Then minimum, maximum and average utilities can be computed for each alternative $A_{i}$ as follows.

If the uncertainty turned out to be against to the assessed alternative, then the minimum utility value is calculated as:

$$
u_{\text {min }}(A)=u\left(G_{1}\right) * g_{1} \%+u\left(G_{2}\right) * g_{2} \%+u\left(G_{3}\right) * g_{3} \%+u\left(G_{1}\right) * g_{1} \%+u\left(G_{2}\right) * g_{2} \%+u\left(G_{1}\right) * g_{1} \%
$$


If the uncertainty turned out to be favorable to the assessed alternative, then the maximum utility value is calculated as:

$$
u_{\max }(A)=u\left(G_{1}\right) *_{1} \%+u\left(G_{2}\right) *_{2} \%+u\left(G_{3}\right) *_{3} \%+u\left(G_{2}\right) *_{2} \%+u\left(G_{3}\right) *_{3} \%+u\left(G_{3}\right) *_{3} \%
$$

The final value is the average of the maximum and minimum utility values. The final values arranged in sorted order gives the ranks of preference.

This process has to be repeated for all the assessments provided by all the assessors. A simplified version of IER is Laplace Evidential Reasoning ${ }^{[15]}$ that is introduced by the author. It is based on Laplace Principle of Insufficient Reason that can be explored for further usage and applicability.

\section{CONCLUSION}

A comprehensive model for decision-making incorporating uncertainty is presented. The assessments collected using interval scale with probabilities distributed across the intervals best describe the incorporation of uncertainty. The aggregated values generated using Interval Evidential Reasoning and the study of different aggregation algorithms is expected to seek the attention of researchers in the field of decision-making.

\section{REFERENCES}

1. Raanan, L. and Orna, S. 1997. "Coping with Uncertainty: A Naturalistic Decision Making Analysis," Organisational Behaviour and Human Decision Processes, 69: 149-163.

2. Galbraith, J. 1973. “Designing Complex Organizations,”. Addison-Wesley, Reading: MA.

3. James G March and Johan P Olsen, 1976. “Ambiguity and Choice in Organisations,” Bergen, Norway, Universitiesforlaget.

4. Keeney, R.L. 1972. "Utility Functions for Multiattributed Consequences," Management Science, 18: 276-287.

5. Eom, H. 1989. "The Current State of Multiple Criteria Decision Support Systems," Human Systems Management, 8(2): 113-119.

6. Stevens, S.S. 1946. "On the Theory of Scales of Measurement," Published in Science, New Series, 103(2684): 677-680, 1946.

7. Persis, V. and Vinaya Babu, A. 2013. "Comparison of requirements prioritization techniques employing different scales of measurement," ACM SIGSOFT Software Engineering Notes, 38(4).

8. Saaty, T. 1980. "The Analytic Hierarchy Process: Planning, Priority Setting, Resource Allocation," McGraw-Hill.

9. Seaver, D. 1976. "Assessment of Group Preferences and Group Uncertainty for Decision-Making," University of Southern California Social Science Research Institute, Research Report. pp. 76-4: 1-61. 
10. Dong-Ling Xu, Jian-Bo Yang, Ying-Ming Wang, 2006. "The evidential reasoning approach for multiattribute decision analysis under interval uncertainty," European Journal of Operational Research, 174: 1914-1943, Elsevier.

11. Wang, Y-M. and Yang, J.B. et al. 2006. "The evidential reasoning approach for multiple attribute decision analysis using interval belief degrees," European Journal of Operational Research, 175: 35-66.

12. Jian Bo Yang and Dong Ling Xu, 2002. "On the Evidential Reasoning Algorithm for Multiple Attribute Decision Analysis Under Uncertainty," IEEE Transactions on Systems, Man and Cybernetics, 32(3): 289-304.

13. Thomas L Saaty, 2008. "Decision Making with the Analytic Hierarchy Process," Int. J. Services, 1(1).

14. Yang, J.B., Deng, M. and Xu, D.L. 2001. "Nonlinear regression to estimate both weights and utilities via evidential reasoning for MADM," In Proceedings of $5^{\text {th }}$ International Conference on Optimization Techniques and Applications, Hong Kong, Dec 15-17.

15. Persis, V. and Vinaya Babu, A 2017. "Study of aggregation algorithms for aggregating imprecise software requirements' priorities, European Journal of Operational Research. 259(3): 1191-1199. 